\title{
Estrategias complejas en la resolución de problemas matemáticos contextualizados
}

\section{Complex strategies in solving contextualized mathematical problems}

Resumen: El objetivo complejo de investigación es desmitificar las falsas herencias de la resolución de problemas en Matemáticas que han dado muchos problemas, e ir a estrategias complejas, bajo la luz de la teoría de la complejidad en la palabra de Dios que alumbra. De esta manera, el pensamiento complejo y el razonamiento lógico matemático se integran para la resolución de problemas contextualizados. Se realiza la indagación con el transmétodo la deconstrucción rizomática transcompleja, se ubica en la línea de investigación titulada: Educación Matemática Decolonial Transcompleja. Indagación realizada en el marco del Postdoctorado en Educación Matemática: Pensamiento, Religaje y Construcción de Emergentes Formativos en la Transmodernidad, Universidad Nacional Experimental de Yaracuy, Venezuela. Luego de una deconstrucción de la resolución de problemas como metodología de enseñanza, se dan características de las estrategias complejas en cuanto a: "sistema, circularidad, dialógica, causalidad compleja, interacciones, círculos polirrelacionales y religación (MORÍN, CIURANA y MOTTA, 2002).

Palabras clave: Enseñanza de la Matemática. Estrategias complejas. Resolución de problemas. Re-ligar. Decolonialidad planetaria.

Abstract: The complex objective of research is to demystify the false inheritances of problem solving in mathematics that have given many problems, and to go to complex strategies, in the light of the theory of complexity in the word of God that illuminates. In this way, complex thinking and mathematical logical reasoning are integrated for solving contextualized problems. The investigation is carried out with the trans-complex rhizomatic deconstruction method, it is located in the line of research titled: Transcomplex Decolonial Mathematical Education. Inquiry carried out within the framework of the Postdoctorate in Mathematical Education: Thought, Religging and Construction of Training Emergencies in Transmodernity, National Experimental University of Yaracuy, Venezuela. After a deconstruction of problem solving as a teaching methodology, characteristics of complex strategies are given in terms of: "system, circularity, dialogic, complex causality, interactions, polyrelational circles and religation (MORÍN, CIURANA \& MOTTA, 2002).

Keywords: Mathematics Education. Complex strategies. Problem solving. Re-bind. Planetary decoloniality.

\section{Rizoma. Introito y transmetodología de la investigación}

En la presente investigación, se destaca el compromiso con la investigación mi humildad y sacrificio de amor día a día con la sabiduría de Dios, para la autora es un asunto 


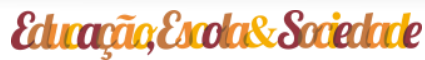

de entrega, de dedicación, seriedad absoluta en lo que hace; no porque para mi sea más fácil hacerlo; sino pues le convoca la enseñanza de la Matemática como un problemática a resolver; en estos momentos de metamorfosis; se entiende que la formación docente, la del ciudadano también pasa por la metamorfosis en los momentos actuales mundiales. Para Dios no hay nada imposible. Esta expresión implica el compromiso de la autora con su hacer empeñado con Dios; pero no quita cientificidad ni perdida del sentido y objetivo complejo de la investigación. Se quiere hacer ver que en los métodos tradicionales las subjetividades de los autores no podían estan en las investigaciones; sin embargo acá el uso de los transmétodo permite tales manifestaciones con el uso de los transmétodo; que ratifico no quita cientificidad a la indagación.

Ubicado en el transparadigma transcomplejo la resolución de problemas debe estar inmersa en la complejidad y transdisciplinariedad; que se debe, primeramente, ir al hacer deconstructivo de lo conocido de la resolución de problemas para desenmascar la problemática en la resolución de problemas, ¿que sucede con los problemas de la resolución de problemas como metodología de Educación Matemática? Sino existe la deconstrucción se corre el riesgo de cometer los mismos errores o peores.

Se destaca que cuando se habla de resolución de problemas matemáticos de refiere a dicha metodología como forma de enseñanza. Es de clarificar que en Matemática siempre se quiere en la mayoría de las veces resolver problemas, por toda la historia de la humanidad. Acompañando de Plaza e González (2019), entre otros autores de la historia y filosofía de la Matemática en esta investigación se hace, primeramente una breve revisión de las principales premisas para resolver problemas de Matemática. Los autores que investigan normalmente comienzan en el año 1500 su revisión. La autora va antes en el tiempo. El objetivo complejo de investigación es desmitificar las falsas creencias de la resolución de problemas en Matemáticas que han dado muchos problemas, e ir a estrategias complejas, bajo la luz de la teoría de la complejidad en la palabra de Dios que alumbra. De esta manera, el pensamiento complejo y el razonamiento lógico matemático se integran para la resolución de problemas contextualizados.

Para la realización de la investigación se uso la deconstrucción rizomática como transmétodo de la investigación, enmarcada en la transmodernidad y transcomplejidad; que publica Rodríguez (2019a). Referimos que los rizomas investigativos se realizan con un transmétodo, que es complejo y transdisciplinar; esto es transcomplejo. Esta transmetódica es "concepto que envuelve lo metametódico; constituye y postula un ascenso en el pensamiento (...) se observa un rebasamiento de los tradicionales trasfondos paradigmáticos (...) es un ejercicio de reflexión donde se repiensa permanentemente el statu quo de las 


\section{Edurañäa,EscolaßSaciedace}

ciências" (ZAÁ, 2017, p. 91).

Desde luego, dejan estas últimas de erigir como las eruditas del saber para abrirse a lo olvidado y desmitificado, a lo que no se pone en escena en la resolución de problemas. La deconstrucción "como transmétodo sólo es posible en la transcomplejidad bajo el proyecto transmoderno" (RODRÍGUEZ, 2019a, p. 13). Se analizan emergentes de la resolución de problemas en su devenir histórico en la línea de investigación titulada: Educación Matemática Decolonial Transcompleja. La deconstrucción como transmétodo de investigación, "es libre al máximo, anti-dogmática, no tiene ninguna transmetodología fija, su objetivo es debilitar el pensamiento filosófico occidental, destruir las concepciones colonizantes en todas sus formas y significados (RODRÍGUEZ, 2019a, p. 43).

Así, este transmétodo, busca involucrarse en procesos develadores encubiertos en discursos, prácticas, acciones y discursos impresos de la resolución de problemas, para con esto descomponerlos, desarticularlos y re-ligarlos con un sentido otro en la temporalidad que nos ocupa, desde una visión de hologramática, del todo y sus partes, de las partes y el todos en un mecanismo permanente de bucle recursivo (MORÍN, 1996), acto que es considerado por la autora como "proceso descolonizador" (RODRÍGUEZ, 2019a, p. 54), que lleva a los sujetos que la utilizan a la creación de transepistemologías que invitan a la emergencia de movimientos emancipatorios; que van de la mano con la enseñanza de la Matemática y la resolución de problemas.

Acá se hace "desenmascaramiento del pensamiento occidental, de la modernidad, los ideales tradicionales impuestos de la educación y sus representaciones sociales" (RODRÍGUEZ, 2019a, p. 7) en tanto la resolución de problemas, como metodología ha estado permeando la Educación Matemática en general, en una desmotivación que ha llevado memorísticamente a la enseñanza algorítmica incambiable de la Matemática; desprendida de la lógica dialéctica para primeramente desde el re-ligar en la Matemática aprender a pensar, un arte recatado del legado antiguo, de los griegos entre otros; que lleven a un viraje de lo que significa enseñar y aprender Matemáticas.

Devine el nombre de rizoma en la presente indagación de la nominación de la Biología, en la tradicionalidad, en comienzo de rizoma en la indagación; no es una casualidad, es la profunda transdisciplinariedad que no le atribuye apellidos consensuado dueños en las concepciones o nominaciones: la denominación de rizoma en la estructura de la investigación, "se trata de una anti-genealogía que rompe con las estructuras estáticas divisorias de presentar las indagaciones en las que las partes se dividen indisolublemente en 


\section{Edurañãa,Escola\&Sociedace}

un ir si un venir" (RODRÍGUEZ, 2019a, p. 4).

Intentamos construir bases sólidas a las mesetas en la deconstrucción como transmétodo, sólo posible en la decolonialidad planetaria, en la transmodernidad; por ello no es extraño ir a Occidente y discurrir con investigadores planetarios. Se quiere pensar profundo, ver en donde el pensar disyuntivo impidió la metacognición de alto nivel en la resolución de problemas, George Papy matemático entrevistado lo afirma que las Matemáticas nos vinculan con el Ser, con la realidad "constato que las Matemáticas tocan estructuras psicológicas profundas (...) podemos decir que el dominio del lenguaje matemático ejerce un efecto terapêutico" (PÉREZ, 1980, p. 45).

Con la deconstrucción se esta en un "ir y venir que va a la criticidad; pero también a la reconstrucción" (RODRÍGUEZ, 2019a, p. 9) del arte de pensar como un lema descartiano que incite a pensar profundo con la Matemática. En un re-ligar, des-ligando primeramente (RODRÍGUEZ, 2019a) que convoca a pensar matemáticamente, la deconstrucción es reconstructiva y va conjugar y analizar en rizomas: devenir de la resolución de problemas en la Matemática, estrategias complejas en la resolución de problemas de la Matemática y rizoma conclusivo a modo de aperturas en las estrategias complejas en la resolución de problemas en la Matemática., en la con-formación del ciudadano (RODRÍGUEZ, 2013).

\section{Rizoma. Devenir de la resolución de problemas en la Matemática}

En la antigua Matemática griega existían tres problemas clásicos de Matemática: la cuadratura del círculo, la duplicación del cubo, la trisección del ángulo, eso lo explica Crespo (1996) en la clásica: Tekhne, Revista de Ingeniería. Esos problemas se planteaban usando utilizando únicamente una regla sin marcas; es decir sin medidas y un compás; por cierto, dicha regla y compas los denominaba Platón instrumentos divinos.

Las rectas y los círculos eran las denominadas curvas perfectas; más sin embargo cualquier otra figura usada tenían un grado de impureza; la recta la asemejaba Platón con la rectitud y el buen comportamiento; no es extraño que Aristóteles publicará su libro Ética, en donde la Matemática interviene. Y es la misma Grecia donde la pereza intelectual es uno de los pecados capitales, concepción más tarde de los judíos.

Es de subrayar que Sócrates veía las Matemáticas como instrumento indispensable de la formación intelectual. Esta ciencia, en términos de Sócrates, al igual que los debates contradictorios que tanto atraían a la juventud, debía servir para formar mentes, formar 


\section{Edurañâ,Escolar Sociedace}

espíritus. Para Platón, dicha ciencia cumple una función propedéutica de magnitud distintiva, pues deben servir de introducción al estudio de la filosofía y la propedéutica del conocimiento (SIGARRETA, RODRÍGUEZ y RUESGA, 2006).

No se ha de olvidar que en obra la República, Platón muestra su evidente fascinación y formación en Matemática, plantea que si se quiere desarrollar la inteligencia es preciso proceder como se hace en Geometría, por medio de problemas (SIGARRETA, RODRÍGUEZ y RUESGA, 2006). Sócrates fue capaz de aislar la noción de resolver problemas para someterla a estudios; a pesar de su idea de que solamente podemos conocernos a nosotros mismos, hay que destacar en el elementos metacognitivos importantes, y estudiados en la actualidad, como factores que intervienen en la solución de problemas (SCHOENFELD, 1987).

Por otro lado, hablar de la resolución de problemas es ir al el estudio de la historia y filosofía de la Matemática; la excelentísima historia, que se cuenta como ninguna otra historia; se cuenta haciendo Matemática; es la oportunidad de rectificar desde nuestra formación el re-ligaje oportuno y certero. En el legendario libro titulado: Elementos de historia de las Matemáticas de Bourbaki Nicolas se cuenta, hablando de pandemia; que en el año 430 a.C. una epidemia de fiebre tifoidea mató a la cuarta parte de la población de Atenas; incluido a Pericles; les invito a los docentes de matemáticos y matemáticos a discernir lo siguiente: ¿Qué tiene que ver dicha epidemia y la muerte de Pericles con los tres problemas clásicos de la Matemática nombrados anteriormente? ¿Cómo intervino Platón en ese problema?

Por cierto, Nicolás Bourbaki se llama el matemático que nunca existió (GORDÓN, 2011); Bombal (2011) cuenta tal azaña; de como Halmos (1957) dice de Nicolás Bourbaki que es:

Es griego, su nacionalidad francesa y su historia es curiosa. Es uno de los matemáticos más influyentes del siglo XX (...) Sus trabajos se leen y citan extensamente en todo el mundo. (...) Tiene fervientes partidarios y acérrimos detractores en cualquier grupo de matemáticos que se reúna. El hecho más extraño sobre él, sin embargo, es que no existe. (BOMBAL, 2011, p. 75)

Tal asunto misterioso reside en el hecho que, narra Bombal (2011) que a mediado de 1930 hasta 1970 nace un grupo de matemáticos, la mayoría franceses, que bajo el seudónimo colectivo de Nicolás Bourbaki “influyó decisivamente en el desarrollo y la evolución de la Matemática contemporánea" (BOMBAL, 2011, p. 77). El grupo se funda en Francia en protesta a la situación de las Matemáticas allí, estaba integrado por brillantes matemáticos de 


\section{Edurañãa,Escola\&Sociedace}

20 a 30 años (BOMBAL, 2011).

Es importante la transcendencia en la resolución de problemas de dicho grupo notable de Matemáticas. Con el terrible error de imponer a jóvenes universitarios que apenas comienzan la Matemática de la talla de estos matemáticos; de allí hay un asunto peyorativo al grupo; pero no es culpa del grupo de Bourbaki; sino de quienes usaban la Matemática como ejercicio de soslayación para descartar seres humanos en el aula. Matemática de alto nivel impuesta en la denominada Matemática Moderna a estudiantes universitarios que comenzaban a estudiar Matemáticas.

Es esencial destacar Jean Piaget y su relación con algunos miembros del grupo de Bourbaki, especialmente con Jean Dieudonné, Jean Piaget:

Llegó a la conclusión de que existe una correspondencia muy estrecha entre los procesos del desarrollo psicológico espontáneo de la mente infantil, por medio de la cual el niño interactúa con el mundo, y las nociones de estructuras madre de las que hablaban los bourbakistas. Así pues, las estructuras mentales que permiten que los seres humanos puedan pensar de forma lógica tomarían como modelo estructuras matemáticas. (BOMBAL, 2011, p. 91)

Como agregado esencial, la sal que los docentes de Matemática en Educación Inicial deben de conocer que, cada niño conoce, por ejemplo; el número como una estructura mental, mediante una aptitud natural al pensar, en vez de aprenderla del exterior, o impuesta en una numeración consecutiva de repetición. No hace falta enseñar la adición a los niños de temprana edad, sino explorar en sus juegos lo que ellos ya saben; darles innegables guías que les permitirán desarrollar su razonamiento numérico (PIAGET, 1920). Cuando se refiere a sus juegos, se habla de juegos de los niños de su hogar y no los impuestos por el docente.

En el asunto del pensamiento complejo sobre los estudios de Jean Piaget, Edgar Morín declaró ser partidario del constructivismo piagetiano, pero acota en Jean Piaget, que su concepción es "necesarias fuerzas organizacionales complejas innatas para que haya fuertes aptitudes para conocer y aprender" (MORÍN, 1996, p. 27). Manifiesta el autor la clara aceptación que todos podemos aprender, y que es necesaria la clara concepción compleja a considerar.

Barberousse (2008) estudia las coincidencias y desencuentros del pensamiento complejo con las teorías de Jean Piaget. Así, Piaget (1967) encontró la autorregulación como punto de partida para la comprensión de los procesos cognitivos y afirma que "los procesos cognitivos aparecen simultáneamente como los resultados de la auto-regulación organísmica, 
cuyos mecanismos esenciales reflejan, y como los órganos más diferenciados de esta regulación en el seno de las interacciones con el exterior" (PIAGET, 1967, p. 49).

Morín (1996) integró, de Jean Piaget de autorregulación, el principio de autoecoorganización, aclaremos que su idea central giró en torno al hecho de que "el sujeto como observador/perceptuador no puede concebirse aislado del objeto estudiado, y se le debe considerar en y por su relación coorganizadora con su ambiente y entorno en el marco de un pensamiento ecologizante" (BARBEROUSSE, 2008, p. 108).

Regresando al recorrido de la resolución de problemas, Plaza y González (2019), y afirmo que como jPienso y entonces soy! parafraseando a Rene Descartes, en el año de 1637 en el Discurso del Método, expone por medio de cuatro reglas simples, los pasos mediante los cuales se debe articular cualquier proceso investigativo. Afirma que la intuición y la deducción son las principales operaciones de la mente, permitiendo que éstas en el método indiquen la mejor manera de seguir el orden, es decir, en guiar las proposiciones de lo más simple a lo más complejo (PLAZA y GONZÁLEZ, 2019). En la regla 1, no se debe asumir nada verdadero, sino se tiene la evidencia respectiva. En la segunda regla, aduce que todo problema debe dividirse en tantas partes como sea posible, porque cuando se disgrega lo complejo en sus partes más sencillas, el intelecto se hace más claro. La regla No. 3, conduce bajo un orden de dificultad los pensamientos. Y en la última regla, se llevan a cabo las enumeraciones y revisiones tan complejas y normales que permita la seguridad de no haber obviado algún detalle (DESCARTES, 1999). Es de hacer notar que en la segunda regla Descartes no plantea que no se debe volver a la conexión de las partes del problema con el todo del problema; creo que en lo personal las reglas de su método han sido mal interpretadas.

Más tarde, en 1908 Henri Poincaré a partir del pensamiento evolutivo, plantea 4 fases para la solución crítica y creativa de los problemas de Matemática, denominados: saturación, incubación, inspiración y verificación (POINCARÉ, 1994). Basándose en la obra de Poincaré (1994) el francés Jacques Hadamard, explica las siguientes fases en la resolución de problemas; documentación, preparación, incubación, iluminación, verificación y conclusión, (HADAMARD, 1945). En el mismo año de 1945 se dan los estudios del matemático húngaro George Pólya, quien es considerado el pionero en "una metodología para la resolución de problemas de Matemática, mediante un enfoque heurístico y con una perspectiva global y no exclusivamente Matemática” (PLAZA y GONZÁLEZ, 2019, p. 171).

Sin embargo, Contreras (1987) afirma que George Pólya en Zurich en 1931en la Sociedad Suiza de Profesores de Matemáticas, anuncia en una conferencia que pretendía 


\section{Edurañãa,Escolas Sociedade}

presentar un nuevo método de enseñanza de la Matemática, titulado: como buscar la solución de un problemas de Matemáticas (POLYA, 1934), "su línea de trabajo que podía llamarse hoy método de enseñanza por resolución de problemas, preocupa a un gran número de profesores de la comunidad de educadores de Matemáticas” (CONTRERAS, 1987, p. 49).

Las razones que explica Contreras (1987) es que como estrategia metodológica un problema no es un recurso mediante el cual pretendemos una automatización rutinaria de un procedimiento, ni la asimilación de determinados algoritmos por repetitivas aplicaciones mecánicas de éstos. Hay que considerar que el mismo George Polya afirma poner en pie un plan, “concebir la idea de la solución, ello no tiene nada de fácil. Hace falta, para lograrlo, el concurso de toda una serie de circunstancias: conocimientos ya adquiridos, buenos hábitos de pensamiento, concentración, y lo que es más, buena suerte” (PÓLYA, 1976, p. 33).

Luego de George Polya, todos los estudios en materia metodológica desde la resolución de problemas para enseñar Matemática devienen de sus avances. Los pasos de George Polya se resumen en cuatro (4) fases o etapas: comprender el problema, desarrollar una estrategia, ejecutar el plan y examinar la solución obtenida. Es de entender que Allan Schöenfeld que redefine los pasos de George Polya termino concluyendo que el uso de estrategias heurísticas no resulta exitoso y encomienda desplegar estrategias para problemas específicos (SCHÖENFELD, 1993).

Ha de considerar para los frenéticos de los pasos, para los cautivadores de un algoritmo incambiable en resolver problemas que es esencial la consideración compleja en la enseñanza de la Matemática, tomando en cuenta que para ir a resolver problemas hay que explorar cada uno de estos aspectos, aquí si con el frenesí e intensidad mayor, esto es: el conocimiento de base, o lo que el discente conoce de la teoría conceptual Matemática referida al problema, donde es muy importante el lenguaje y la semiótica; las estrategias complejas de resolución de problemas sin que estas sean fijas e incambiables (tomando en cuenta que toda estrategia compleja lleva a una acción, más adelante se detallan dichas estrategias); los aspectos metacognitivos; los aspectos afectivos y el sistema de creencias aunado a la comunidad de práctica; esto es el aspecto cultural, cotidiano del discente (SCHÖENFELD, 1992).

Es de hacer notar que estos pasos incambiables de los que habla el autor anterior han creado y colaborado en las creencias y concepciones negativas hacia la resolución de problemas em Matemática y son producto del razonamiento y entendimiento; incluyen creencias, significados, preferencias, conceptos, proposiciones, reglas, e imágenes mentales 
que influyen en lo que se percibe y en los procesos de razonamiento que se realizan (LOPEZLEYTON, ALDANA y ERAZO, 2018). Es vital el razonar criticamente.

El pensamiento crítico, como parte vital del pensamiento complejo es esencial en la resolución de problemas. Ilustremos este punto con Martin Gardner, en su libro titulado: Matemática para divertirse, un paseo por las diversas ramas de la Matemática a través de más de 50 problemas de ingenio.

En tal obra se plantea el siguiente acertijo: titulado: sin tiempo para la escuela:

Pero no tengo tiempo para la escuela, explicaba Eddie al director. Duermo ocho horas diarias que, sumadas, dan 122 días por año, suponiendo que cada día es de 24 horas. No hay clases los sábados ni los domingos, que suman 104 días por año. Tenemos 60 días de vacaciones de verano. Necesito tres horas diarias para comer, esto es más de 45 días al año. $\mathrm{Y}$ necesito al menos dos horas diarias de recreación, lo que suma más de 30 días al año. Eddie escribió estas cifras mientras hablaba, después sumó todos los días. La suma daba 361. Ya ve, continuó Eddie; eso me deja tan sólo cuatro días para estar enfermo y en cama, y ni siquiera he tomado en cuenta los siete feriados escolares que tenemos cada año. El director se rascó la cabeza. Algo no anda bien aquí, murmuró. Pero por más que se esforzó, no pudo encontrar nada equivocado en las cifras de Eddie. Los profesores de matemática pudieran mostrar a sus estudiantes: ¿Puedes explicar dónde está el error? (GARDNER, 2008, p. 73)

Pensar en ese acertijo es tener un pensamiento crítico; para ello se necesita de la conceptualización, de la teoría, del manejo de la semiótica, del lenguaje; pero también necesita de la madurez de la conceptualización del discente. Lanzarse si ello a los pasos para resolver un problema es inmersionar en una piscina sin agua. Por ello, el propósito de la Matemática ante todo es profundizar el pensamiento, es el desarrollo metacognitivo; el pensar profundo; el juego y comprensión de los símbolos, la motivación; de allí va una preparación fabulosa a la hora de resolver problemas.

El desarrollo metacognitivo, alude a la necesidad de toda una consideración compleja en la resolución de problemas, el estudio de la comprensión cabal de los enunciados com la semiótica del lenguaje, los datos que da el problema entendidos adecuadamente no sólo datos numericos, la relación que se establece entre los datos que permean el problema y la premisa que se debe resolver, es de consideración permanente los procesos de razonamiento que el discente con sus y habilidades como son la síntesis, abstracción, concreción, ejemplo, el análisis, la visualización, la argumentación, la fluidez del lenguaje, la audacia, la autonomía (ARTEAGA-MARTÍNEZ, MACÍAS e PIZARRO, 2020). Es toda una estructura compleja que no debe ser desmitificada en la enseñanza.

En los primeros niveles el nivel lúdico debe ser casi el 100\% en la enseñanza de la 


\section{Edurañãa,Escola\&Sociedace}

Matemática, ya lo explica el propio matemático divertido, afirma:

El elemento lúdico que hace recreativa a la matemática recreativa puede tomar muchas formas: un problema para resolver, un juego competitivo, un truco de magia, una paradoja, una falacia o simplemente matemática con alguna vuelta curiosa o divertida. ¿Son estos ejemplos de matemática pura o aplicada? Es difícil decirlo. En un sentido la matemática recreativa es matemática pura, incontaminada de utilidad. En otro sentido, es matemática aplicada, ya que responde a la necesidad humana de jugar. (GARDNER, 1984, p. 124).

En cuanto a la contextualización de los problemas de Matemática sobre todo en los primeros niveles; la Educación Matemática Realista es un enfoque que considera que la Matemática en las aulas de clases debe ser orientada desde la cotidianidad, con una actividad intensa entre docente y discente, y también darle la oportunidad al estudiante de reinventar las Matemáticas. Actualmente el realismo ha distado muy lejos de la realidad de los estudiantes y de la escuela, en muchas ocasiones en las clases se presentan problemas bien remotos de sus verdaderas realidades. $\mathrm{Al}$ respecto el deber ser de esta temática afirma que

realizar una reflexión sobre la realidad como referente para nuestra actuación docente, prestando especial atención a las falsas realidades tan presentes aún en nuestra enseñanza e indicando las características deseables del realismo educativo. Gran parte del tiempo dedicado a la enseñanza de la matemática se dedica a la resolución de ejercicios rutinarios alejados de la vida cotidiana. (ALSINA, 2007, p. 85)

Se pueden revisar otras publicaciones de Claudi Alsina, que inventan diferentes formas de enseñar Matemáticas, la Matemática desde el corazón, Matemática hermosa se enseña con el corazón, hecho que ha manifestado y justificado en sus artículos y disertaciones en numerosos eventos.

El recorrido realizado en la resolución de problemas, no es estricto pero si develo la carencia en la resolución de problemas la crisis. Por ello, en lo que deviene se describe de manera teórica las estrategias complejas recomendadas en la resolución de problemas.

\section{Rizoma. Estrategias complejas en la resolución de problemas de la Matemática}

Volviendo al acertijo anterior que se planto del matemático Gardner, ¿que les parece si arman una estrategia compleja para resolver el acertijo anterior?; para ello hay que pensar complejamente; este en si mismo es una estrategia compleja. Tenemos que considerar que toda "nuestra enseñanza tiende al programa mientras que la vida nos pide estrategia" 
(MORÍN, 2000, p. 80); la resolución de problemas no necesita cualquier estrategia. Una estrategia compleja requiere: sistema, circularidad, dialógica, causalidad compleja, interacciones, círculos polirrelacionales y religación (ARROYAVE, 2003; MORÍN, CIURANA e MOTTA, 2002).

Expliquemos cada uno de estos requerimientos que posee la estrategia compleja en la resolución de problemas; con el sistema "se puede concebir el sistema como unidad global organizada de interrelaciones entre elementos, acciones o individuos" (MORÍN, 1992, p. 124), así es importante la comunicación entre todas las partes del problemas es importante; así mismo comprendiendo el todo del el. La noción de que el problema de Matemática es un sistema estriba también en que "la interrelación de los elementos y la unidad global constituida por estos elementos en interacción. (..) se puede concebir el sistema como unidad global organizada de interrelaciones entre elementos, acciones o individuos (MORÍN, 1986, p. 124).

Elproblema de Matemática no puede estar aislado en los primeros niveles educativos, de la realidad cultura, juegos y cotidianidad del discente; esto es clave en los primeros niveles a fin de evitar traumas psicológicos a los niños y niñas, en la entrevista al matemático George Papy afirma

Los niños o individuos que han estado bloqueados para aprender
matemáticas, han estado bloqueados también en su personalidad. Un niño
que no aprendió matemáticas se siente disminuido en sí mismo como
individuo. Se puede hablar, pues, de una relación profunda entre el
conocimiento matemático y la personalidade. (PÉREZ, 1980, p. 4)

Sigue afirmando el entrevistado que esto no ocurre del mismo modo con otras disciplinas; y que por el contrario el dominio del lenguaje matemático ejerce un efecto terapéutico.

Con la circularidad en las estrategias complejas "entrevemos la posibilidad de transformar los círculos viciosos en ciclos virtuosos, que lleguen a ser reflexivos y generadores de un pensamiento complejo" (MORÍN, 1992, p. 32); se trata que en la resolución de problemas nos podemos encerrar en errores que permean el problema; así es importante que ahora estos se transforme en ciclos para ir desmitificando los errores y la comprensión total del problema. Los errores que bordean la compresión del problema a medida que se siguen cometiendo se ascienden a la comprensión del problema en cuanto a su lenguaje y manera de entrever el problema. Muchas veces el problema de comprender el problema no estriba en el problema en si; sino en el lenguaje que este comunica o no con le lenguaje de compresión del discente. 


\section{Edurañăa,Escolad Sociedace}

Con la dialógica en la estrategia compleja para la resolución de problemas, permitir "asumir racionalmente la inseparabilidad de unas nociones contradictorias para concebir un mismo fenómeno complejo" (MORÍN, 2002, p. 126) es primero no olvidar que el problema que se asume es complejo; pues todas sus partes unidas forman un todo; no se trata de la complicación del problema; es la totalidad comunicada del problema. Desde luego, con la dialógica ponemos a comunicarse las partes del problema; pues "la dialógica introduce pluralidades, separaciones, oposiciones, retroactividades y calor; el calor le trae agitaciones y desordenes" (MORÍN, 1992, p. 39); todo ello debe asumirse como parte del problema.

La causalidad compleja en la estrategia compleja "crea lo improbable, en este sentido, no concierne ya solamente a cuerpos aislados o poblaciones, sino también a seres individuales que interactúan con su entorno" (MORÍN, 1986, p. 308); por ello eso que llamamos casual en el problema debemos entenderlo como una causalidad entre sus partes, en el todo. Sigue explicando Edgar Morín, que para "comprender cualquier cosa en la vida, la sociedad, el individuo, hay que recurrir al juego complejo de las causalidades internas y externas" (MORÍN, 1986, p. 308). Ese juego de las causalidades explica como el docente en el medio de ella debe recurrir a consideraciones del ser humano, en esa condición humana, que antes separaba en el aula.

Por ello, debe recurrir al sentir del discente, ¿Qué le agrada, que rechaza?, ¿por qué es conveniente comenzar con un problema de tal nivel, en consideración a los conocimientos previos del discente?; debe recurrir el docente a cambios en la condiciones iniciales del problema, a su simbología y manera de presentarlo, en su esquematización para que sea comprendido por el discente; el juego de palabras; como interactúa con él; comprende el dicente lo que el problema necesita para ser resuelto; lo entiende en su necesidad como juego mental del problema.

De las interacciones en la estrategia compleja:

Las interacciones: 1. suponen elementos, seres u objetos materiales que pueden encontrarse; 2. suponen condiciones de encuentro, es decir, agitación, turbulencia, flujos contrarios; 3. obedecen a determinaciones/constreñimientos que dependen de la naturaleza de los elementos, objetos o seres que se encuentran; 4 . En ciertas condiciones se convierten en interrelaciones (asociaciones, uniones, combinaciones, comunicación), es decir, dan lugar a nuevos fenómenos de organización. (MORÍN, 1986, p. 69).

Estas características de las estrategias complejas esta relacionada con la causalidad; así como las otras características que el dan la condición de compleja a dichas estrategias.

El juego de las asociaciones, uniones, combinaciones entre otros accionares deben estar en condición 


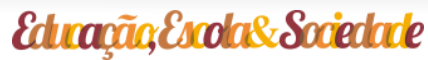

especial de la estrategia; pero debe ser del agrado y compresión metacognitiva del discente; así cada uno reacciona y tarda en comprender más que otro de sus compañeros; entonces las estrategias complejas necesitan entrar en el juego de la diversidad de comprensión, emotividad, creencias y afectividad del discente; pero también de su mundo, cotidianidad y cultura. Si las estrategias lejos de acercar a juego de las ideas a los discentes los separan; sin duda no son las adecuadas; sin importar si el docente les agrada y considera que son las apropiadas. La comprensión primerísima, factibilidad, agrado en el discente es lo importante.

El contexto cultural, e intercultural no debe ser desmitificado em la resolución de problemas; "incorporar algunas de las estrategias personales de los estudiantes durante la enseñanza formal en el aula de clases de Matemáticas, como una forma de practicar la interculturalidad y promover la participación de los estudiantes" (GARCÍA-GARCÍA, 2019, p. 205). Es el contexto cultural-cotidiano de alto potencial motivador a considerar en la resolución de problemas en la Matemática; asi la formación docente al particular debe mirarse com cuidado y ser actualizada en todo momento; estudiando cada diversidad de ser humano a educar.

Es urgente, en los primeros niveles educativos el asunto de la contextualización de los problemas de la Matemática y ello forma parte de las estrategias complejas; "el desarrollo de la aptitud para contextualizar y globalizar los saberes se convierte en un imperativo de educación" (MORÍN, 2000, p. 31). Allí interviene lo que veníamos hablando en la deconstrucción de las falsas realidades explicadas por Claudi Alsina en la resolución de problemas. Es urgente en la resolución de problemas "de principios organizativos que permitan unir los saberes y darles sentido" (MORÍN, 2000, p. 25). De los círculos polirrelacionales

Las interrelaciones entre los diversos componentes de los subsistemas y del sistema educativo complejo, en general, están dadas como en un círculo polirrelacional, donde los componentes, aunque, deben ser definidos a la vez, en y por sus caracteres originales, en y con las interrelaciones de las que participan, en y con la perspectiva de la organización en la que están dispuestos, en y con la perspectiva del todo en el que se integram. (MORÍN, 1996, p. 366)

Se hace enfasis em la contextualización, dado que la interacción com la cotidianidad del discente es urgente, el sentido en los primeros niveles educativos de la Matemática, "lo primero que tiene que hacer un estudiante es conceptualizar, cuando tiene claro el concepto entonces una forma de verificarlo es enfrentarse a diferentes tipos de ejercicios y problemas en contextos situados de aprendizaje" (LÓPEZ-LEYTON, ALDANA y ERAZO, 2019, p. 16) 


\section{Edurañãa,Escola\&Sociedace}

Muchas maneras o casi todas las estrategias a la hora de resolver problemas están basadas en el modo escalera del conocer de la Matemática; en tanto sus conocimientos previos. Si no ha entrado en el primer escaló, el docente debe desmitificar el hecho en tanto echar la cumpla a los otros niveles educativos y comenzar desde el primer escalón, a fin de que el ascenso sea a pasos certeros seguros. Para ello, tendrá que explorar en al dialógica de primer orden con cada discente.

En estos círculos polirrelacionales al que Edgar Morín hace referencia en las estrategias complejas los elementos del problema de Matemática deben ser definidos, y desde la historia y filosófica de la Matemática las interrelaciones se deben integrar al problema en una especie de motivación; en la que el discente cobre interés por la problemática que aborda el problema; y de como otros en la historia lo abordaron. Es de tomar en cuenta, y la Matemática tiene por excelencia lo policultural, que nuestras sociedades contemporáneas son "policulturales y comportan su(s) cultura(s), su cultura nacional (que eventualmente integra culturas etnorregionales), una "cultura de masas" sincrética que vehiculan los grandes medios, la cultura científica y, por ultimo, aquello que hace dos siglos era considerado como la cultura" (MORÍN, 1992, p. 70).

Es necesario que por si mismo los círculos en las estrategias complejas son circularidades, que permiten, "abrir la posibilidad de un método que, al hacer interactuar los términos que se remiten unos a otros, se haría productivo, a través de estos procesos y cambios, de un conocimiento complejo que comporte su propia reflexividad" (MORÍN, 1986, p. 32). Esta circularidad nos aporta metodologías complejas, llevadas de la mano de los transmetodos denominados así por la autora pues trascienden los métodos cerrados no complejos tradicionales; que en este caso apartarán otras mesetas de salidas a los problemas de Matemática.

En tal sentido, el ser docente de Matemática es un ejercicio comprometido con la condición humana del discente, el debe ser conocedor del sujeto bumano de su condición, de su ambivalente, de sus motivaciones; en las Matemáticas es esencial; las creencias negativas y afectividad disminuida le gana a la mejor estrategia; pues ninguna de ella es buena sino atiende, en primera instancia al sujeto aprendiz; "al individuo hay que considerarlo a la vez como totalmente dependiente y verdaderamente autónomo. (..) es el centro necesario de toda acción, interacción, producción, reproducción de la poli-organización viviente" (MORÍN, 1993, p. 319).

Es importante considerar en esas estrategias complejas para que le sirven al discente 
si podrán con ellos legar a procesos metacogntivos de alto nivel para la formación de su persona, en tanto el desarrollo de un pensar profundo; la enseñanza de la Matemática "debe contribuir a la autoformación de la persona (aprender y asumir la condición humana, aprender a vivir) y aprender a convertirse en un ciudadano. Un ciudadano, en una democracia, se define por su solidaridad y su responsabilidad” (MORÍN, 2000, p. 83).

La religación su noción es deconstruida en la complejidad y asi en la estrategia compleja, pues la religación trae un vacio conceptual dándole una "naturaleza sustantiva a lo que solo era concebido adjetivamente, y dándole un carácter activo a este sustantivo. "Religado" es pasivo, "religante" es participante, "religación" es activante. Se puede hablar de "desligación” para el opuesto de "religación"” (MORÍN, 2004, p. 235). Así, es menester des-ligar las nociones reduccionistas de resolver problemas, para ir a re-ligar ideas novedosas aparentemente incomunicadas con el problema que le dan otras vías de entendimiento; aquí debe yacer la creatividad, la innovación; el uso de herramientas tecnológicas con la apertura clara del docente que se des-liga de su vieja formación para crear procesos conviviales de enseñanza.

En el re-ligaje es importante considerar la enseñanza de la Matemática dependiendo el nivel educativo, hemos de interrogarnos si los problemas de Matemáticas escolares de primaria, ¿son sólo problemas para el aula? (CHAMOSO et al., 2013). Este nivel educativo como la Educación Básica es esencial para acercar a la vida del discente la Matemática, ellas pueden hacerse cercanas a los discentes y se debe aludir a situaciones más concretas, sin dejando de lado el pasar a la abstracción en niveles adecuados; la concreción va en términos de objetos, lugares y personajes, para que pudieran ser entendidas por los estudiantes como situaciones más representativas de sus vidas.

Hay mucho que explorar en las estrategias complejas en la resolución de problemas de Matemáticas, ya no este como metodología de enseñar Matemáticas; entendemos que no existe tal metodología única, impuesta aferrada a pasos irrestrictitos e incambiables. La estrategia compleja se impone siempre que sobreviene lo inesperado o lo incierto, es decir, desde que aparece un problema importante que asumimos complejo. Toda estrategia, en cualquier dominio que sea, tiene conciencia de la apuesta a lo incierto; lleva la acción que es estrategia compleja perse. La estrategia permite, a partir de una decisión inicial, imaginar un cierto número de escenarios para la acción (MORÍN, 1996).

El pensamiento complejo es un desafío, que invita al diseño y la puesta en práctica de estrategias complejas que conduzcan a salir de las crisis en la que se encuentra sumergidas la enseñanza de la Matemática. Ciertamente las estrategias son necesarias para salir de la crisis, pero se deben abandonar des- 


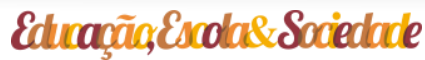

ligando los vicios y las viejas posturas, las soluciones viejas y elaborar nuevas soluciones. La enseñanza de la Matemática debe ser compleja dejando de ser el sistema cerrado impenetrable. La enseñanza de la Matemática desde la complejidad parte de un pensamiento disidente, flexible, reflexivo, porque esta comprende que todo lo que marca una referencia en la bistoria nace de lo inesperado.

Partiendo del principio de lo inesperado y reconociendo que la estrategia necesita de la complejidad, porque en condiciones normales en donde no existen las perturbaciones se puede ser autómata, programado es posible, el algoritmo es esencial si es comprendido; pero hay que recordar que cada problema a resolver tiene su propia diversidad pero hay que recordar que la estrategia deviene de lo inesperado o lo incierto de los escenarios de la enseñanza, de la comprensión de discente en discente, de su experiencia de vida y su afectividad por el proceso con-formativo.

Por último, en lo que culmina y estudio que apenas comienza debemos considerar la característica transdisciplinaria de las estrategias complejas en la resolución de problemas. Y se debe cohabitar con el conocimiento transdiciplinar: para poder conseguir estrategias para la convivencia de los saberes de la Matemática (RODRÍGUEZ, 2019b). Donde se ha de tener en cuenta: que la comprensión del otro como modo de aprendizaje desde su complejización es una estrategia que en el accionar, estrategias que requieren reformular la disciplina Matemática, deconstruyendo, reconstruyendo y redefiniendo sus propias estructuras lógicas en cada caso y la manera de comunicarse; también accionar en el ejercicio de poder de quienes portan la disciplina como un cintillo de verdad. Los saberes soterrados eminentemente culturales como apertura en lo científico y viceversa es una estrategia de primera línea; la diada individuo-tolerancia para admitir lo complejo como diversidad y unidad como estrategia; entre otras que Rodríguez (2019b) detalla.

La investigación continua em futuras investigaciones; en la línea de investigación titulada: Educación Matemática Decolonial Transcompleja; con indagaciones de problemas de Matemática contextualizado, de Educación Primaria Venezolana donde se erijan algunas estrategias complejas para la solución, considerando las características de las estrategias complejas: sistema, circularidad, dialógica, causalidad compleja, interacciones, círculos polirrelacionales y religación (ARROYAVE, 2003; MORÍN, CIURANA y MOTTA, 2002). Adaptando las particularidades del problema, las necesidades del discente con dichas características. Siempre en la consideración decolonial planetaria, transdisciplinar y compleja que no excluye, que abraza en un diálogo de saberes lo execrado. 


\section{Eduraçãa,EsodarSociedtade}

\section{Rizoma conclusivo a modo de aperturas en las estrategias complejas de la resolución de problemas}

Con la deconstrucción como transmétodo, en forma envolvente, incisiva se há deconstruido la resolución de problemas en la Matemática y se analizarán teóricamente características de las estrategias complejas a la luz de la teoría de la complejidad. La construcción no es definitiva y se retroactua en cada momento en la enseñanza; son esencias de un comienzo. El recorrido deconstructivo de la resolución de problemas no es estricto y devine en cada momento a considerar.

Se rescata en la cabalidad del objetivo complejo propuesto, las esencias de las estrategias complejas a considerar en la resolución de problemas en la Matemática, estas características de: sistema, circularidad, dialógica, causalidad compleja, interacciones, círculos polirrelacionales y religación deben ser anidadas en el momento, diversidad de pensamientos, cultura y cotidianidad de los discentes en los procesos educativos; ello precisa un ejercicio metacognitivo de alto nivel en el docente; el debe alcanzar dichos recursivos pensamientos sobre lo que es educar en la resolución de problemas a fin de comprender a cabalidad los procesos dialógicos necesarios para su comunicabilidad y logro en cada estudiante.

Es imperativo la formación compleja del docente en Matemáticas, pues com estas características de las estrategias complejas puede acudir a una reconstrucción de la historia de la Matemática em el aula, de como fuerón insmiscuyendose los protagonistas en la resolución tradicional de los problemas de Matemática en diferentes niveles de los contenidos. Es motivo de la continuación de la línea de investigación donde se insmiscuye esta investigación la: Educación Matemática Decolonial Transcompleja de estúdios más profundos en dicho tema.

El camino del conocer es una meseta hermosa que vale la pena siempre transitar. Como matemático, como mi ejercicio favorito de pensar profundo, siempre afirmo: cuando estudio matemáticas mis pensamientos se eleva hasta el universo, y sus similitudes de perfección con esté me hacen entender el poema más bello que de la misma vida se hace.

En la enseñanza de la Matemática decolonial, donde se cobra una Matemática viva com mente, cuerpo y corazón se dan vivencias que cobran la afectividad en los sujetos ya actores dolentes y agentes de cambios em la enseñanza de la Matemática; acudiendo a procesos dialógicos complejos en los discentes. Ya casi al culminar la indagación, em el libro: las Matemáticas del amor y la amistad, el poema número 2 titulado: el tiempo: que es motivo de estudio de la Matemática y resoluciones de problemas 


\section{Edurañãa,Escola\&Sociedace}

Las horas de los días parecen eternas pasan en todas y todos de manera inexplicable; sólo yo me he quedado eternizada en un solo momento en un único suspirar y temo salirme de allí, pues puedo darme cuenta que ya me he ido en ese momento.

Si ese tiempo que a los matemáticos nos tendrá cuan infinito dando buscándolo y cada día se aleja más.

Oh Dios si, el tiempo es relativo no hay duda que Albert Einstein no se equivocó; por eso cuando pienso en el en tan bello momento el tiempo se me va tan rápido y repito y parece eterno cuando te espero; cuando te pienso.

Tiempo de mi vida hasta infinito y en ese regalo de DIOS cumple tu eternidad en mí para pensarte para soñarte, y que cada día en la relatividad de los avatares de la vida pueda mirarte. (RODRÍGUEZ, 2018, p. 15).

Una vez cumplido el objetivo complejo de la investigación y dentro de ella; al final, comienzo y fin del pensamiento profundo que se discierne en el estudio de la Matemática; el máximo nivel de la inteligencia espiritual es la sabiduría que sólo tu Espíritu Santo mi Dios amado, matemático por excelencia magnifico, nos da: "la exposición de tus palabras alumbra; hace entender a los simples" (Salmos 119: 130). Amén. Fe y Fe. Seguimos con DIOS todo y siempre la gloria es para ÉL. Manifestasiones subjetivas de la autora en agradecimiento y fé en Dios que no afectan la cientificidad de la indagación; por el contrario, x'confluyen, en vista de la libertad del transmétodo de investigación usado, a la sabiduría de Dios para el discernimiento adecuado.

\section{Referencias}

ALSINA, Claudi. Si Enrique VIII tuvo 6 Esposas, ¿Cuántas tuvo Enrique IV? El Realismo en Educación Matemática y sus Implicaciones Docentes. Revista Iberoamericana de Educación, Madrid, v. 43, p. 85-101, 2007.

ARROYAVE, Dora. La revolución pedagógica precedida por la revolución del pensamiento: un encuentro entre el pensamiento moriniano y la pedagogía. En Manual de iniciación Pedagógica al Pensamiento Complejo. Ecuador: Publicaciones UNESCO, 2003.

ARTEAGA-MARTÍNEZ, Blanca; MACÍAS, Jesús; PIZARRO, Nohemí. La representación en la resolución de problemas matemáticos: un análisis de estrategias metacognitivas de estudiantes de secundaria. Uniciencia, v. 34, n. 1, p. 263-280, ene./jun. 2020.

BARBEROUSSE, Paulette. Fundamentos teóricos del pensamiento complejo de Edgar Morin. Educare, Heredia, v. 12, n. 2, p. 95-115, 2008.

BOMBAL, Fernando. Nicolás Bourbaki: el matemático que nunca existió. Revista Real Academia Ciencias Exactas Fisicas Naturales, Madrid, v. 105, n. 1, p. 77-98, 2011.

CHAMOSO, José; VICENTE, Santiago; MANCHADO, Eva.; MÚÑEZ, David. Los problemas de matemáticas escolares de primaria, ¿'son solo problemas para el aula? En: 
MORALES, Yuri; RAMIREZ, Alexa (Ed.), Memorias I CEMACYC. Santo Domingo, República Dominicana: CEMACYC, 2013, p. 1-17.

CONTRERAS, Luis. La resolución de problemas, ¿una panacea metodológica? Enseñanza de las ciencias, Madrid, v. 5, n. 1, p. 49-52, 1987.

CRESPO, Luis. Los tres problemas griegos (sin solución). Tekhne Revista de Ingeniería, Caracas, v. 1, p. 71-74, 1996.

DESCARTES, Rene. Discurso del método: seguido de la búsqueda de la verdad mediante la luz natural. Tradución de Víctor Florián. Bogotá: Panamericana Editorial, 1999.

GARCÍA-GARCÍA, Javier. Estrategias en la resolución de problemas algebraicos en um contexto intercultural en el nivel superior. Bolema, Rio Claro, v. 33, n. 63, p. 205-225, 2019.

GARDNER, Martin. Matemática para divertirse, un paseo por las diversas ramas de la Matemática a través de más de 50 problemas de ingenio. Barcelona: Gedisa, 2008.

GARDNER, Martin. Sixth Book of Mathematical Diversions from Scientific American. Chicago: The University of Chicago Press, 1984.

GORDÓN, Fernando. Nicolás Bourbaki: el matemático que nunca existió. Revista Académica Ciencias Exactas Física Naturales, Madrid , v. 105, n. 1, p 77-98, 2011.

HADAMARD, Jacques. An essay on the psychology of invention in the mathematical field. Princeton: Ediciones Princeton University Press, 1945.

HALMOS, Paul. Nicolas Bourbaki. Scientific American, Madrd, v. 196, p. 88-99, 1957.

LÓPEZ-LEYTON, Cristhian; ALDANA, Eliécer; ERAZO, Jhon D. Concepciones de los profesores sobre la resolución de problemas en cálculo diferencial e integral. Logos Ciencia \& Tecnología, Bogotá, v. 10, n. 1, p. 145-157, 2018.

LÓPEZ-LEYTON, Cristhian; ALDANA, Eliécer; ERAZO, Jhon D. El papel de la resolución de problemas para la enseñanza del Cálculo Integral: un estudio de caso. Revista Espacios, Caracas, v. 40, n, 17, p. 12-20, 2019.

MORÍN, Edgar. El Método I: La naturaleza de la Naturaleza. Madrid: Catedra, 1986.

MORÍN, Edgar. El Método II. La vida de la vida. Madrid: Cátedra, 1993.

MORÍN, Edgar. El Método IV: Las ideas. Madrid: Cátedra, 1992.

MORÍN, Edgar. El método VI: Ética. Madrid: Cátedra, 2004.

MORÍN, Edgar. Introducción al Pensamiento Complejo. Barcelona: Gedissa, 1996.

MORÍN, Edgar. La cabeza bien puesta: repensar la reforma, reformar el pensamiento. Bases para una reforma educativa. Buenos Aires: Nueva Visión, 2002.

MORÍN, Edgar. La mente bien ordenada. Madrid: Ediciones Seix Barral, 2000. 


\section{Edurañãa,Escola\&Sociedace}

MORÍN, Edgar; CIURANA, Emilio; MOTTA, Raul. Educar en la era planetária: el pensamiento complejo como método de aprendizaje en el error y la bumana. Bógota: UNESCO, 20002.

PÉREZ, Augusto. Las matemáticas modernas: pedagogía, antropología y política. Entrevista a George Papy. Perfiles Educativos, v. 10, p. 41-46, oct./dic. 1980.

PIAGET, Jean. Lógica y conocimiento científico. París: Gallimard, 1967.

PIAGET, Jean. Psicología del niño. Madrid: Ediciones Morata, 1920.

PLAZA, Luis; GONZÁLEZ, José. Evolución de la resolución de problemas matemáticos: análisis histórico a partir del siglo XVI. Actas Latinoamericana de Matemática Educativa, Ciudad de México, v. 32, n. 2, 168-176, ago. 2019.

POINCARÉ, Henri. Ciencia y método. Buenos Aires: Espasa Calpe, 1944.

POLYA, George. Como buscar la solución de un problema de Matemáticas in Matemática Elemental. Madrid: Editorial Trillas, 1934.

POLYA, George. Mathematical Discovery: On Understanding, Learning and Teaching Problem Solving. New York: Editorial Combined, 1976.

RODRÍGUEZ, Milagros Elena. Cohabitando con el conocimiento transdisciplinar: estrategias para la convivencia de los saberes. Investigación Educativa Duranguense, Durango, v. 11, n. 19 , p. $5-15,2019 b$.

RODRÍGUEZ, Milagros Elena. Deconstrucción: un transmétodo rizomático transcomplejo en la transmodernidad. Sinergias Educativas, Ecuador, v. 4, n. 2, p. 1-13, jul./dic. 2019a.

RODRÍGUEZ, Milagros Elena. La Educación Matemática en la con-formación del ciudadano. TELOS - Revista de Estudios Interdisciplinarios en Ciencias Sociales, Maracaibo, v. 15, n. 2, p. 215-230, 2013.

RODRÍGUEZ, Milagros Elena. Las matemáticas del amor y la amistad. Caracas: Editorial El Perro y la Rana, 2018.

SCHÖENFELD, Alan Henry. Resolución de problemas: elementos para una propuesta en el aprendizaje de la Matemática. Cuadernos de Investigación, México, n. 25, p. 6-8, 1993.

SCHÖENFELD, Alan Henry. A brief and biased bistory of problem solving. Berleley: Ed University of California, 1987.

SCHÖENFELD, Alan Henry. Learning to think mathematically: problem solving, metacognition and sense making in Mathematics. New York: Macmillan, 1992.

SIGARRETA, José: RODRÍGUEZ, Jose; RUESGA, Pilar. La resolución de problemas: una visión histórico-didáctica. Boletín de la Asociación Matemática Venezolana, Caracas, v. 13, n. 1, p. 53-60, 2006.

ZAÁ, José. Pensamiento filosófico transcomplejo. San Joaquín de Turmero: Escriba Escuela de Escritores, 2007. 